\title{
Nuevos materiales ecogénicos y dispositivos de ecoguiado en anestesia regional
}

\author{
J. L. Laguillo Cadenas, J. Fernández Jiménez y F. J. Marques Asin \\ Unidad de Gestión Clínica, Anestesia y Bloque Quirúrgico. Hospital Virgen Macarena. Sevilla
}

Laguillo Cadenas JL, Fernández Jiménez J, Marques Asin FJ. Nuevos materiales ecogénicos y dispositivos de ecoguiado en anestesia regional. Rev Soc Esp Dolor 2013; 20(2): 55-60.

\begin{abstract}
Regional anesthesia and ultrasonography have apparently gone hand in hand in the last decade. The difficulty of visualizing the puncture material in the ultrasound machine during the analgesic or anesthetic techniques is added to that of the nerve structure identification. Different devices and advances on previous materials are appearing so as to facilitate this visualization to the anaesthesiologist. This review aims to gather the information published about these developments.
\end{abstract}

Key words: Anesthesia. Analgesia. Ultrasonography. Needles. Catheters.

\section{RESUMEN}

La anestesia regional y la ecografía parecen ir de la mano en la última década. A la dificultad de la identificación de estructuras nerviosas se añade la de visualizar en el ecógrafo el material de punción conforme se realizan las técnicas analgésicas o anestésicas. La ecogenicidad de agujas y catéteres se debe a la reflexión del ultrasonido en la superficie de Estos. Para incrementar su visualización y facilitar al anestesiólogo la técnica, se han desarrollado nuevos dispositivos y avances en los acabados de estos materiales de punción. El objeto de esta revisión es recopilar de la literatura la información publicada acerca de este desarrollo en materiales de punción para anestesia o analgesia regional.

Palabras clave: Anestesia. Analgesia. Ultrasonografía. Agujas. Catéteres.

Recibido: 02-04-12.

Aceptado: 08-07-12.

\section{INTRODUCCIÓN}

Las primeras referencias de publicaciones en relación a la práctica de la anestesia regional guiada con ecografía son de hace más de 20 años (1), sin embargo, el verdadero auge de estas técnicas ha llegado en estos últimos años gracias a los avances tecnológicos y al desarrollo de sistemas portátiles e imágenes de alta resolución. Este desarrollo abarca al ecógrafo, así como al material empleado en las técnicas locorregionales. En este artículo describimos la situación actual en el desarrollo de agujas, catéteres y dispositivos de ayuda a la punción guiada mediante ecografía.

\section{AGUJAS}

\section{Antecedentes y factores relacionados con la visibilidad de la aguja}

Frente al abanico de ventajas que ofrece el guiado por ecografía en la anestesia regional (2), también se presentan dificultades: precio de equipos portátiles, esterilidad durante el procedimiento o curva de aprendizaje. Este aprendizaje debe realizarse no únicamente en la exploración ecográfica e identificación de estructuras, sino también en lo que es más difícil: el control de la posición de la punta de la aguja durante la realización de la técnica (3). Este último es un elemento esencial para evitar morbilidad al evitar las punciones vasculares, neurales o viscerales inadvertidas. Esto es especialmente relevante en la práctica de anestesia regional en pacientes no colaboradores, que requieren de sedación para su realización y por tanto reduciendo o anulando el guiado por neuroestimulación, como ocurre en la población pediátrica (4).

Aunque la preocupación por mejorar la visualización de la aguja en ecografía intervencionista no es reciente (5), el 
desarrollo en anestesia regional sí está teniendo lugar en los últimos años.

De hecho, Maecken y cols. (6) estudiaron la visibilidad de 12 agujas de neuroestimulación para bloqueo de nervio periférico en condiciones clínicas y obtuvieron una visibilidad considerada como inaceptable en 9 de ellas cuando se insertaban en un tejido animal a $45^{\circ}$ de inclinación, recomendando tan solo 3 de las agujas estudiadas (Pajunk Plexolong, Braun Stimuplex A y D).

La angulación de entrada de la aguja durante el abordaje parece ser un factor crítico en la discriminación de la aguja. La reflexión del haz de ultrasonidos es óptima para la mayor parte de agujas cuando la superficie de la aguja se encuentra a $90^{\circ}$ con respecto al transductor. Sin embargo, conforme el ángulo sea menor, la reflexión y por tanto la visibilidad de la aguja también serán menores (7), surgiendo más diferencias entre modelos y fabricantes en función de sus características por debajo de los $45^{\circ}$ de aproximación. Además, esta verticalización de la aguja suele emplearse buscando objetivos más profundos, asociando fenómenos de atenuación y refracción a través de las distintas interfases y planos tisulares, generándose mayor distorsión y artefactos en consecuencia (8).

El diámetro de la aguja también se ha relacionado con la visibilidad de la aguja. Schafhalter-Zoppoth y cols. (7) encontraron diferencias en la visibilidad en función del calibre de la aguja: a mayor diámetro mayor visibilidad, justificado por la mayor facilidad para alinear el transductor y haz de ultrasonidos con el eje de la aguja. Parece ser que este factor es más relevante en abordajes con angulación cercana a la horizontal, que en aquellos más verticales (9).

La conformación y orientación de la punta de la aguja también puede hacer más identificable a una aguja con respecto a otra: las agujas biseladas se identificarían mejor que las curvadas o cónicas en los abordajes verticales (9). Otros factores que parecen influir en la visión de la aguja serían el acabado y composición química del recubrimiento de la aguja (10), así como la ocupación del canal de la aguja con estilete (7).

\section{Avances y dispositivos comercializados}

Los avances tecnológicos han ido encaminados a incrementar la reflexión del haz de ultrasonidos, de vuelta hacia el transductor. Los radiólogos intervencionistas fueron los primeros interesados en este desarrollo con la finalidad de realizar biopsias ecoguiadas. Sus avances en este campo se han aplicando al material de punción empleado por los anestesiólogos en técnicas regionales, diseñándose agujas más reflectantes, que a su vez pueden también estar aisladas para poder asociar la neuroestimulación (agujas híbridas). Aunque la oferta de productos comienza a ser variada (Fig. 1), los estudios comparativos publicados, empleando agu-

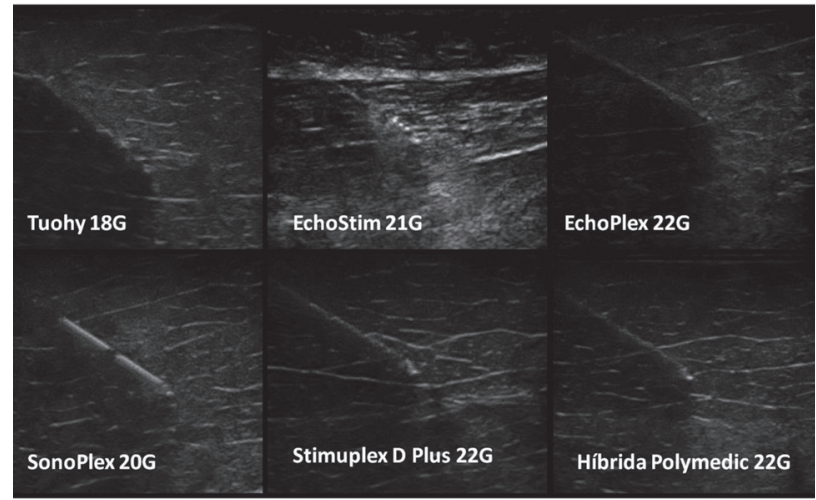

Fig. 1. Visualización de agujas en un phantom animal insertadas a $45^{\circ}$. No ecogénica: Perifix $18 \mathrm{G}$ (Braun). Ecogénica: EchoStim ${ }^{\circledR}$ (Havels, imagen cedida por Dr G. Hocking), Echoplex (Vygon), SonoPlex (Pajunk), Stimuplex ${ }^{\circledR}$ D Plus (Braun), Polyplex US (Polymedic).

jas ecogénicas para anestesia regional, son escasos hasta el momento.

Deam y cols. (11) estudiaron in vitro la modificación en la textura de la superficie del extremo distal de una aguja para bloqueo de nervio periférico (punta cónica 22G) frente a otras tres no modificadas (punta cónica $22 \mathrm{G}$, bisel $30^{\circ}$ $22 \mathrm{G}$ y Tuohy $18 \mathrm{G})$. Como resultado principal encuentran una mayor visibilidad en todos los ángulos para la aguja texturizada, tanto en abordajes en eje corto como en eje largo. La diferencia en visibilidad con las otras agujas era aún mayor en angulaciones cercanas a la vertical, entre $10^{\circ}$ y $20^{\circ}$, señalando que esto pudiera tener relevancia en la práctica de bloqueos profundos como a nivel infraclavicular o del nervio ciático proximal. No aportan más información sobre la tecnología empleada para texturizar la superficie de la aguja.

Edgcombe (9) y Hocking comparan en el cadáver cinco agujas de bloqueo de nervio periférico, tres de ellas con modificaciones para incrementar la ecogenicidad. Establecen como objetivo principal la visualización de la punta de la aguja, obteniendo mejor resultado para dos agujas que enfocaron sus avances en la punta de la aguja, Hakko EchoStim ${ }^{\circledR}$ (Havels, Cincinnati, Ohio) y SonoPlex (Pajunk Medizintechnologie, Geisingen, Alemania).

Hacen énfasis en la visualización de la punta de la aguja, más que en el eje o cuerpo de la misma, para evitar el error de creer estar obteniendo una imagen de la aguja en su integridad, mientras el bisel de la aguja estuviera fuera del campo de visión.

Para esto, la aguja para bloqueo de nervio periférico híbrida EchoStim ${ }^{\circledR}$ (Havel, Hakko Medical, Japón) (12) tiene practicadas tres perforaciones anguladas o cúbicas (CCR-Corner Cube Reflectors) en los 3,5 mm distales de la aguja, permaneciendo la marca distal a 2,5 $\mathrm{mm}$ de la punta del bisel. No está comercializada en Europa de momento. 


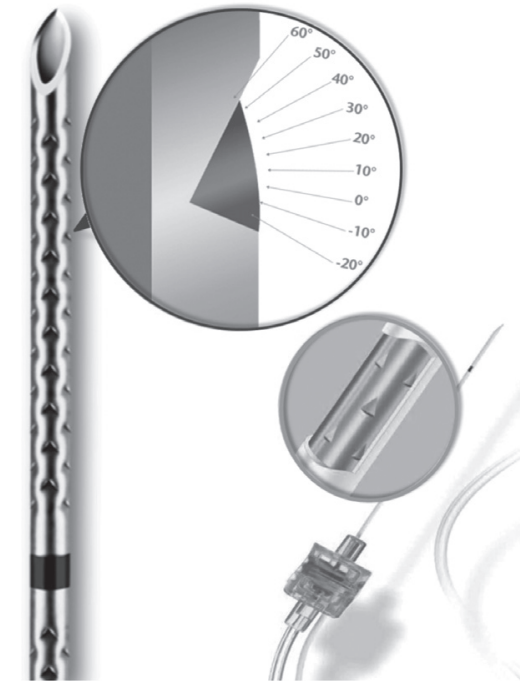

Fig. 2. Representación de impresiones en superficie y recubrimiento aislante. Cortesía de Pajunk y Braun.

Por su parte, la otra aguja híbrida arriba mencionada, SonoPlex (Pajunk Medizintechnologie, Geisingen, Alemania), tiene texturizada su superficie al practicársele también perforaciones cúbicas reflectantes recubiertas por un aislante eléctrico (Nanoline $\left.{ }^{\circledR}\right)$. Estas perforaciones las ha dispuesto en dos secciones de $10 \mathrm{~mm}$ de largo, separadas por un segmento no texturizado de $5 \mathrm{~mm}$. Este patrón simétrico permitiría calcular al operador una situación aproximada de la punta de la aguja, al mismo tiempo que ofrece un mayor tramo reflectante. Recientemente, la aguja Stimuplex® D Plus (Braun, Melsungen, Alemania) ha implementado en su modelo previo (Stimuplex ${ }^{\circledR}$ D) un patrón de marcado por láser en los últimos $20 \mathrm{~mm}$ de la aguja. Está igualmente recubierta por un aislante que permite la electroneuroestimulación a su través. Estas agujas emplean estas marcas o perforaciones, cuya finalidad es exponer una pequeña superficie a una angulación más trasversal al haz de ultrasonidos, facilitando así la reflexión hacia el transductor (Fig. 2).

La aguja Echoplex (Vygon, Aechen, Alemania) ha potenciado la visibilidad mediante el empleo de un aislante ecogénico que expone trazas microscópicas de cristal a lo largo de toda la superficie de la aguja (Fig. 3). Por su parte, el fabricante Polymedic (Temena, Carrieres Sur Seine, France) también dispone de una gama de agujas ecogénicas puras (USB/USC/Poliplex US) e híbridas, imprimiendo rugosidad en el cuerpo metálico de la aguja o recubriéndolas de un polímero aislante ecogénico (Parylene®), respectivamente.

En la literatura, hay escasos estudios comparativos que estudian las diferencias entre estas agujas tratadas ecogénicamente. Blanco y Patel (13) en el que miden, en un modelo in vitro, la visualización de la versión ecogénica de las agujas Pajunk, Vygon, Braun y Polymedic con

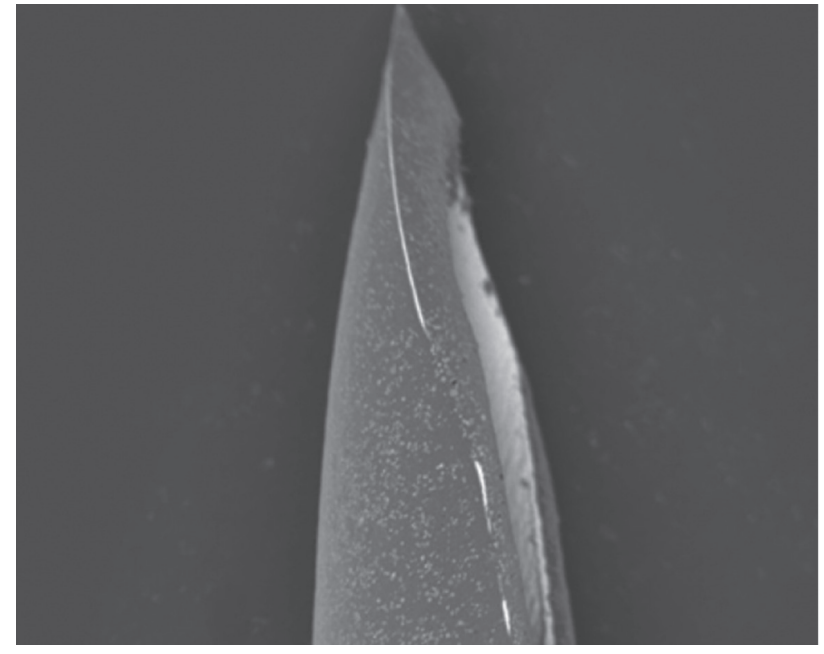

Fig. 3. Microscopía electrónica de barrido: se aprecian microcristales adheridos sobre superficie del cuerpo y bisel. Cortesía de Vygon.

una angulación de 0, 30, 45 y 60 grados respectivamente. Demostraron que las cuatro agujas ecogénicas se visualizaban más intensamente en ángulos pronunciados $\left(45^{\circ} \mathrm{y}\right.$ $\left.60^{\circ}\right)$ que en aquellos más horizontales $\left(10^{\circ}\right)$. Estos resultados son contrarios a los publicados por otros autores, hasta el momento, para agujas no ecogénicas $(6,7,14)$, posiblemente en relación con su tratamiento de superficie frente a agujas no tratadas empleadas en la literatura previa. Obtuvieron mejores resultados de visualización para los modelos tratados de Pajunk y Polymedic.

El empleo de fantom en el aprendizaje de la coordinación mano-aguja ha favorecido su uso también en estos estudios comparativos. En referencia a esto, Hocking (15) insiste en la importancia del tejido adyacente durante la punción para realizar una correcta medición o comparación entre agujas. Para ello estudiaron cuatro agujas ecogénicas diferentes (B-Braun Stimuplex ${ }^{\circledR}$ D plus, Life-Tech y Pajunk ${ }^{\circledR}$ SonoPlex) y no ecogénicas (Pajunk ${ }^{\circledR}$ Uniplex) con cinco modelos diferentes de fantom (agua, blue fantom, gelatina, carne de cerdo, cadáver) y observaron la visibilidad de las agujas en cada uno de ellos a 20 y a 45 grados. Obtuvieron importantes diferencias en visibilidad según el medio o fantom empleado, más destacadas en la angulación de $45^{\circ}$. Resaltan la importancia del empleo de una interfase adecuada a la finalidad de la punción, ya que los resultados obtenidos en medios acuático, sintéticos o animal pueden conducir a falsos positivos en la visualización del cuerpo de la aguja. La simulación en cadáver es la más cercana a la práctica clínica, sin embargo su elevado coste y escasa accesibilidad limitan su empleo. Encontraron resultados de visibilidad similares para las agujas tratadas ecogénicamente en un fantom sintético, aunque destacan le ecogenicidad de la aguja SonoPlex (Pajunk®, Alemania) en el cadáver. 


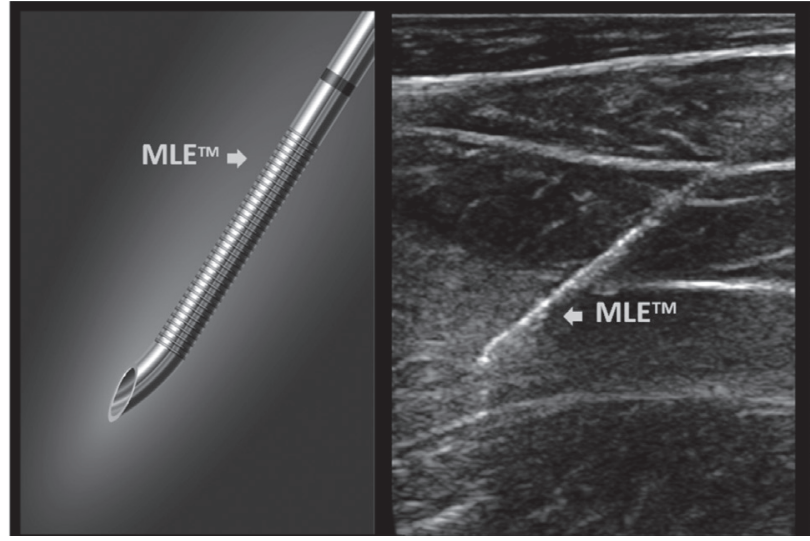

Fig. 4. Aguja con punta de Tuohy marcada en su superficie (EchoTuohy por Havel's). MLE ${ }^{\mathrm{TM}}$ (Micro Laser Etching). Reproducción autorizada.

Las agujas de Tuohy también pueden emplearse en punciones ecoguiadas para técnicas continuas o en punciones únicas (por ejemplo para bloqueo epidural -16- o del plano trasverso abdominal -17-, respectivamente). En contradicción con lo publicado con anterioridad (7), dos de los estudios comparativos reseñados $(9,11)$, obtuvieron una peor visión de la aguja Tuohy $18 \mathrm{G}$ frente a las otras de menor calibre, en abordajes que emplearon una angulación marcada. Esto lo justificaban bien con las diferencias en el acabado mate de la agujas Tuohy empleadas, o bien con la distinta orientación de la punta de estas agujas frente a las biseladas (16). La punta de la aguja EchoTuohy ${ }^{\mathrm{TM}}$ (Havel, Hakko Medical, Japón) mediante un grabado por láser patentado $\left(\mathrm{MLE}^{\mathrm{TM}}\right)$ parece haber incrementado la visibilidad de la misma sin haber modificado su diámetro interno, permitiendo así el avance de catéteres a su través. No está comercializada en Europa de momento (Fig. 4).

No podemos afirmar que la aguja que aporte una mayor visibilidad sea la más recomendable, dado que no hay estudios comparativos publicados sobre eficacia clínica. Para definir la aguja ideal serían varias las características que habrían de considerarse (Tabla I).

\section{CATÉTERES}

El ecoguiado de catéteres en anestesia ha tenido lugar en distintos escenarios, al permitir la visualización de su avance o el emplazamiento final de la punta del catéter. No obstante, emplear la visualización ecográfica durante la práctica de un bloqueo continuo de nervio periférico (BCNP) reviste cierta dificultad. A su salida por el extremo de la aguja, el catéter de BCNP no tiene porqué seguir una trayectoria definida, ni mantener por tanto una conformación rectilínea. Esto hace que la visualización ecográfica del avance del catéter en el plano ecográfico sea difícilmen-
TABLA I. CARACTERÍSTICAS DE LA AGUJA IDEAL PARA ECOGRAFÍA

1. Visibilidad óptima, especialmente de la punta de la aguja

2. Visualización adecuada independiente de la angulación

3. Apta para todo tipo de tejidos

4. Escasa generación de artefactos

5. Sombra acústica no significativa

6. Elevado contraste con el tejido

7. Fácil discriminación del extremo de la aguja

te alcanzable. Además, la mayor parte de los anestesiólogos que emplean ecografía en los BNP exploran los nervios trasversalmente, por lo que en un avance longitudinal al nervio del catéter, únicamente visualizan un corte trasversal del mismo. Debido a esto, la inserción y avance del catéter se realiza prácticamente a ciegas, habitualmente a corta distancia desde el extremo de la aguja. A este respecto, los trabajos publicados han comunicado métodos indirectos para la localización del extremo del catéter: bien asociando neuroestimulación, bien empleando el Doppler color durante la administración de suero o anestésico (18), o bien administrando anestésico local agitado que genere burbujas que actúan como contraste hiperecogénico (19). No obstante, Koscielnak-Nielsen y cols. (20) comunicaron una serie de casos en la que exploraron y guiaron con éxito la introducción de catéteres para BNP continuo de forma longitudinal. De esta forma, pudieron emplazar el extremo del catéter a la altura deseada, así como observar la distribución del anestésico local. Para ello emplearon un catéter Perifix ${ }^{\circledR}$ ONE (Braun, Melsungen, Alemania), que fabricado mediante una doble capa de poliuretano y poliamida proporciona una mayor opacidad y rigidez que los catéteres epidurales convencionales.

En anestesiología, el desarrollo de material ecogénico ha sido enfocado inicialmente hacia las agujas, a pesar de ser conocida la relación que el revestimiento guarda con la reflexión de ultrasonidos (10) y del empleo de catéteres ecogénicos por otras especialidades (21). El catéter neuroestimulante para BCNP SilverStim ${ }^{\text {SM }}$ (Vygon, Aachen, Alemania) está recubierto en toda su longitud (salvo los 3 $\mathrm{mm}$ del extremo) de una impregnación de plata (bajo un aislante de polietileno) que actúa como conductor eléctrico y reflectante ultrasónico. También Polymedic, en su set Echo-Polyplex US Plus, dispone de un catéter 20G que refieren como ecogénico (por incluir un fiador espiroideo metálico que aporta rigidez y engrosamiento de paredes del catéter). No hay trabajos ni experiencias comunicadas con los mismos. 


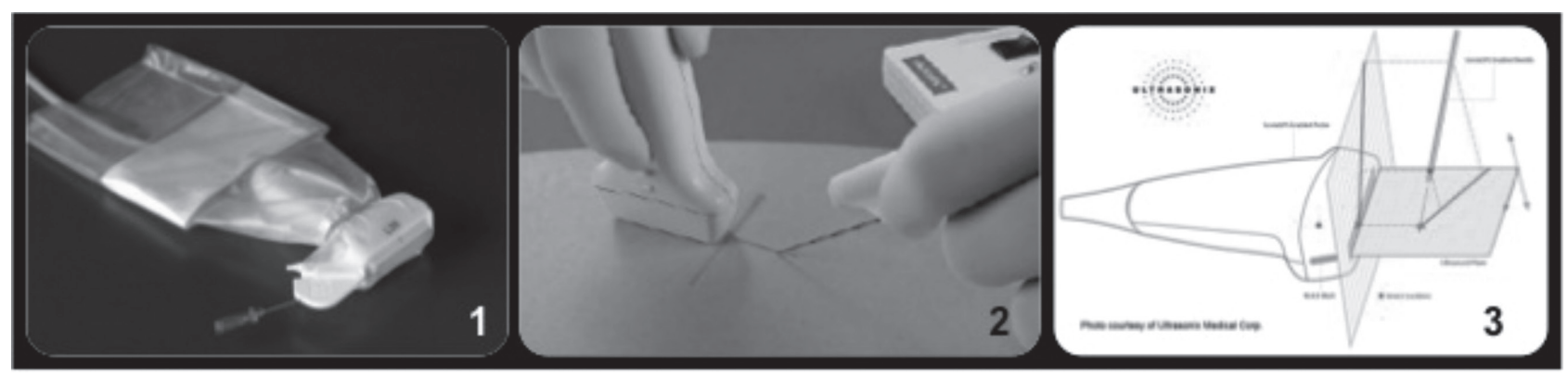

Fig. 5. Nuevos dispositivos de ayuda para la punción: 1- Guías desechables estériles (fabricante CIVCO; imagen cedida desde MAC, Bilbao, España). 2- Sonopointer (Pajunk, Alemania). 3- Guiado tridimensional por GPS (imagen cortesía de SonixGPS, Canadá). Reproducción autorizada.

El aumento de la ecogenicidad en los catéteres también podría suponer una importante aportación en seguridad y eficacia en técnicas neuroaxiales: en la población pediátrica facilitaría la colocación de catéteres en el segmento metamérico óptimo a nivel epidural, pudiendo objetivarse los frecuentes acodamientos tras su inserción a ciegas a nivel caudal (22).

Empleando otro desarrollo previo en radiología intervencionista (ColorMark®), Klein y cols. $(23,24)$ describen la utilización de un dispositivo piezoeléctrico asociado a una aguja y catéter durante un abordaje continuo a nivel ciático en el cadáver. Dicho dispositivo genera una oscilación a través del cuerpo de la aguja o de un estilete metálico flexible insertado en el catéter, cuya intensidad es creciente en dirección al extremo de la aguja o catéter. Estas oscilaciones son captadas por el ecógrafo en modo doppler, sirviendo por tanto como medio de apoyo a la visualización del cuerpo y extremo del catéter. No obstante, muchos ecógrafos pierden definición cuando pasan a modo doppler, hasta el punto de hacer imposible la discriminación del nervio, lo que limitaría el uso de esta técnica (25).

\section{DISPOSITIVOS DE AYUDA A LA PUNCIÓN}

De forma similar a los avances que tuvieron lugar en las agujas, el desarrollo de dispositivos de ayuda a la punción ecoguiada en otras áreas médicas podría emplearse en anestesia regional (Fig. 5).

- Guías para punción: se fijan al transductor y permiten dirigir el avance de la aguja, asegurando una dirección prefijada $(8)^{8}$. En la obtención de biopsias, se ha comparado el uso de guías para punción frente a la técnica de mano libre (26), resultando en punciones más rápidas y eficaces con las guías. Su uso en anestesia regional aún no está muy extendido por el dinamismo de la técnica, al requerir redireccionamiento cuando pretendemos rodear el nervio de solución anestésica. Recientemente se han diseñado estériles y desechables, no requiriendo de procesos de esterilización.

- Guiado por láser: la alineación del haz del ultrasonidos con la aguja en plano puede facilitarse mediante una emisión láser a nivel de la superficie de punción, al mismo tiempo que permite una técnica de mano libre. Tsui Lo describe un prototipo acoplado al transductor (27), si bien en su desarrollo comercial SonoPointer® (Pajunk Medizintechnologie, Geisingen, Alemania) lo han acoplado a la aguja.

- Guiado por GPS/3D: mediante un sistema GPS podría programarse el recorrido y el destino final de la aguja, introduciendo las coordenadas en tres dimensiones. Un sensor electromagnético colocado en la punta de la aguja informa de su posición en tiempo real, así como el recorrido que va a seguir en abordaje largo o corto (28). La empresa UltraSonix (Richmond, Canada) comercializa este dispositivo, SonixGPS, y agujas adaptadas para BNP.

\section{CONCLUSIONES}

El desarrollo del material especifico para anestesia regional ecoguiada está iniciándose. La literatura publicada y la información disponible facilitan pocos datos sobre el mismo, por lo que será necesario evaluar las aportaciones de estas innovaciones en la práctica de la anestesia regional. Para esto serían necesarios trabajos prospectivos y revisiones actualizadas a los distintos dispositivos existentes en el mercado.

\section{Agradecimientos y conflictos de interés}

Agradecemos al Dr. Hocking, así como Havel's Inc (Ohio, Estados Unidos.), CIVCO (Iowa, Estados Unidos), Ultrasonix (Richmond, Canada), Vygon (Aechen, Alemania), Braun (Melsungen, Alemania) y Pajunk (Geisingen, 
Alemania) por la cesión de las imágenes empleadas en este texto.

Ninguno de los autores mantiene o ha mantenido relaciones económicas o personales con las casas comerciales que se mencionan en la presente revisión, salvo las derivadas de la obtención de las figuras reseñadas.

\section{CORRESPONDENCIA}

José Luis Laguillo Cadenas

C/ Beatriz de Suabia ${ }^{\circ} 103$

41005 Sevilla

Telf. 955008305

joseluislaguillo@yahoo.es

\section{BIBLIOGRAFÍA}

1. Kapral S, Krafft P, Eibenberger K, et al. Ultrasound-guided supraclavicular approach for regional anesthesia of the brachial plexus. Anesth Analg 1994;78:507-13.

2. Neal JM, Brull R, Chan VW, et al. The ASRA evidence-based medicine assessment of ultrasound-guided regional anesthesia and pain medicine: Executive summary. Reg Anesth Pain Med 2010;35:S1-9.

3. Sites BD, Spence BC, Gallagher JD, et al. Characterizing novice behavior associated with learning ultrasound-guided peripheral regional anesthesia. Reg Anesth Pain Med 2007;32:107-15.

4. Tsui B, Suresh S. Ultrasound imaging for regional anesthesia in infants, children, and adolescents: a review of current literature and its application in the practice of extremity and trunk blocks. Anesthesiology 2010;112:473-92.

5. Laine HR, Rainio J. An inexpensive method of improving visualisation of the needle tip in fine needle aspiration biopsy (FNAB). Ann Chir Gynaecol 1993;82:43-5.

6. Maecken T, Zenz M, Grau T. Ultrasound characteristics of needles for regional anesthesia. Reg Anesth Pain Med 2007;32:440-7.

7. Schafhalter-Zoppoth I, McCulloch CE, Gray AT. Ultrasound visibility of needles used for regional nerve block: an in vitro study. Reg Anesth Pain Med 2004;29:480-8.

8. Chapman GA, Johnson D, Bodenham AR. Visualisation of needle position using ultrasonography. Anaesthesia 2006; 61:148-58.

9. Edgcombe H, Hocking G. Sonographic identification of needle tip by specialists and novices: a blinded comparison of 5 regional block needles in fresh human cadavers. Reg Anesth Pain Med 2010;35:207-11.

10. McGahan JP. Laboratory assessment of ultrasonic needle and catheter visualization. J Ultrasound Med 1986;5:373-7.
11. Deam RK, Kluger R, Barrington MJ, et al. Investigation of a new echogenic needle for use with ultrasound peripheral nerve blocks. Anaesth Intensive Care 2007;35:582-6.

12. Takayama W, Yasumura R, Kaneko T, et al. [Novel echogenic needle for ultrasound-guided peripheral nerve block "Hakko type CCR"]. Masui 2009;58:503-7.

13. Blanco R. Echogenicity of needles: a comparative in vitro study. Cir May Amb 2010;15:113-6.

14. Nichols K, Wright LB, Spencer T, et al. Changes in ultrasonographic echogenicity and visibility of needles with changes in angles of insonation. J Vasc Interv Radiol 2003;14:1553-7.

15. Hocking G, Hebard S, Mitchell $\mathrm{CH}$. A review of the benefits and pitfalls of phantoms in ultrasound-guided regional anesthesia. Reg Anesth Pain Med 2011;36:162-70.

16. Chan V. Ultrasound Imaging for Regional Anesthesia: a practical guide booklet. Toronto; 2008.

17. Awad IT, Chan V. Ultrasound imaging of peripheral nerves: a need for a new trend. Reg Anesth Pain Med 2005;30:321-3.

18. Dhir S, Ganapathy S. Use of ultrasound guidance and contrast enhancement: a study of continuous infraclavicular brachial plexus approach. Acta Anaesthesiol Scand 2008;52:338-42.

19. Swenson JD, Davis JJ, DeCou JA. A novel approach for assessing catheter position after ultrasound-guided placement of continuous interscalene block. Anesth Analg 2008;106:1015-6.

20. Koscielniak-Nielsen ZJ, Rasmussen H, Hesselbjerg L. Longaxis ultrasound imaging of the nerves and advancement of perineural catheters under direct vision: a preliminary report of four cases. Reg Anesth Pain Med 2008;33:477-82.

21. Coroleu B, Barri PN, Carreras O, et al. Effect of using an echogenic catheter for ultrasound-guided embryo transfer in an IVF programme: a prospective, randomized, controlled study. Hum Reprod 2006;21:1809-15.

22. Tsui BC, Suresh S. Ultrasound imaging for regional anesthesia in infants, children, and adolescents: a review of current literature and its application in the practice of neuraxial blocks. Anesthesiology 2010;112:719-28.

23. Klein SM, Fronheiser MP, Reach J, et al. Piezoelectric vibrating needle and catheter for enhancing ultrasound-guided peripheral nerve blocks. Anesth Analg 2007;105:1858-60.

24. Marhofer P, Harrop-Griffiths W, Kettner SC, et al. Fifteen years of ultrasound guidance in regional anaesthesia: part 1 . Br J Anaesth;104:538-46.

25. Chin KJ, Perlas A, Chan VW, et al. Needle visualization in ultrasound-guided regional anesthesia: challenges and solutions. Reg Anesth Pain Med 2008;33:532-44.

26. Phal PM, Brooks DM, Wolfe R. Sonographically guided biopsy of focal lesions: a comparison of freehand and probeguided techniques using a phantom. AJR Am J Roentgenol 2005;184:1652-6.

27. Tsui BC. Facilitating needle alignment in-plane to an ultrasound beam using a portable laser unit. Reg Anesth Pain Med 2007;32:84-8.

28. Wagner S. Tracking the tip-Improving Accuracy for needle biopsies. Radiology Today 2010;11:30. 